\title{
From sticky-hard-sphere to Lennard-Jones-type clusters
}

\author{
Lukas Trombach, ${ }^{1}$ Robert S. Hoy, ${ }^{2}$ David J. Wales, ${ }^{3}$ and Peter Schwerdtfeger ${ }^{1,4, *}$ \\ ${ }^{1}$ Centre for Theoretical Chemistry and Physics, New Zealand Institute for Advanced Study, Massey University Auckland, \\ Private Bag 102904, 0632 Auckland, New Zealand \\ ${ }^{2}$ Department of Physics, University of South Florida, Tampa, Florida 33620, USA \\ ${ }^{3}$ University Chemical Laboratories, Lensfield Road, Cambridge CB2 1EW, United Kingdom \\ ${ }^{4}$ Centre for Advanced Study (CAS) at the Norwegian Academy of Science and Letters, Drammensveien 78, NO-0271 Oslo, Norway
}

(Received 1 February 2018; published 24 April 2018)

\begin{abstract}
A relation $\mathcal{M}_{\mathrm{SHS} \rightarrow \mathrm{LJ}}$ between the set of nonisomorphic sticky-hard-sphere clusters $\mathcal{M}_{\mathrm{SHS}}$ and the sets of local energy minima $\mathcal{M}_{L J}$ of the $(m, n)$-Lennard-Jones potential $V_{m n}^{\mathrm{LJ}}(r)=\frac{\varepsilon}{n-m}\left[m r^{-n}-n r^{-m}\right]$ is established. The number of nonisomorphic stable clusters depends strongly and nontrivially on both $m$ and $n$ and increases exponentially with increasing cluster size $N$ for $N \gtrsim 10$. While the map from $\mathcal{M}_{\mathrm{SHS}} \rightarrow \mathcal{M}_{\mathrm{SHS} \rightarrow \mathrm{LJ}}$ is noninjective and nonsurjective, the number of Lennard-Jones structures missing from the map is relatively small for cluster sizes up to $N=13$, and most of the missing structures correspond to energetically unfavorable minima even for fairly low $(m, n)$. Furthermore, even the softest Lennard-Jones potential predicts that the coordination of 13 spheres around a central sphere is problematic (the Gregory-Newton problem). A more realistic extended Lennard-Jones potential chosen from coupled-cluster calculations for a rare gas dimer leads to a substantial increase in the number of nonisomorphic clusters, even though the potential curve is very similar to a $(6,12)$-Lennard-Jones potential.
\end{abstract}

DOI: 10.1103/PhysRevE.97.043309

\section{INTRODUCTION}

The nucleation of atoms and molecules in the gas phase, or liquid, to the solid state is still an active research field [1-8]. Rowland noted in 1949 that "The gap between theory and the experimental approaches to nucleation has been too wide" and "the subject [nucleation] is still in the alchemical stage" [9]. More than half a century later, despite all the advancements made in cluster physics, "there is still a large gap between experiment and theory" as Unwin noted [10].

The underlying reason for this rather slow progress is that cluster formation is a dynamic process, and fully characterizing the corresponding high-dimensional potential energy landscape is typically an NP-hard problem since there are (presumably) exponentially many local minima at any given temperature and pressure [1,11-16]. Moreover, phase transitions between different morphologies as a function of size $N$ usually occur where $N$ is too large for an accurate quantumtheoretical treatment [17-21]. For example, Krainyukova experimentally studied the growth of argon clusters [22] and found that small, initially icosahedral clusters transform into anti-Mackay clusters for $N>2000$ and finally into the closed packed fcc or hep structures at $N>10^{5}$ atoms, in qualitative agreement with theoretical predictions using Lennard-Jones (LJ)-type potentials [2,23,24]. The notorious rare gas problem was solved only very recently by accurate relativistic quantum methods, correctly predicting a slight preference of the fcc over the hcp phase due to phonon dispersion [25].

\footnotetext{
*p.a.schwerdtfeger@massey.ac.nz
}

Simple models often have to be used to simulate cluster growth and nucleation [26-29]. The simplest model potentials that can be applied to theoretical studies of atomic cluster formation are "HCR-SRA" potentials with isotropic hardcore-like repulsive and short-range-attractive interactions [30]. The simplest HCR-SRA potential is the "sticky-hard-sphere" (SHS) potential [31]

$$
V_{\mathrm{SHS}}(r)= \begin{cases}\infty, & r<r_{s} \\ -\varepsilon, & r=r_{s}, \\ 0, & r>r_{s}\end{cases}
$$

where $r_{s}$ and $\varepsilon$ can be arbitrarily set to 1 (unit sphere and reduced units, respectively). Equation (1) can be used as a perturbative basis for finite-ranged HCR-SRA potentials [32,33]. Since sticky hard spheres are impenetrable and their energy $E=-N_{c} \varepsilon$ is a function only of the number of interparticle contacts $N_{c}$, SHS cluster structure and energetics can be uniquely mapped to their adjacency matrices $\bar{A}$, where $N_{c}=$ $\sum_{i<j}^{N} A_{i j}$. This mapping allows them to be exactly characterized via complete enumeration [34-36]; recent studies have identified all mechanically stable SHS clusters for $N \leqslant 14$ and putatively complete sets for $N \leqslant 19$ [34-39]. Note, however, that different SHS structures can have the same adjacency matrix for $N \geqslant 14$ [38], and the mapping is therefore only surjective.

From the Gregory-Newton kissing-number argument, proved in 1953 by Schütte and van der Waerden [40], no sphere can be surrounded by more than 12 spheres of equal radius [41]. For small clusters, graph-theoretic arguments dictate $\max \left(N_{c}\right) \leqslant N(N-1) / 2$. Thus a loose bound on the maximum 
contact number $N_{c}(N)$ is

$$
N_{c}^{\max }(N) \leqslant \min \{N(N-1) / 2, f(N)\}
$$

with $f(N)=6 N$. This upper bound has been tightened several times, most recently by Bezdek and Reid [42] to

$$
f(N)=6 N-3(18)^{1 / 3} \pi^{-2 / 3} N^{2 / 3} .
$$

In Refs. [37,38] it was shown that $N_{c}^{\max }(N)=\{6,9,12$, $15,18,21,25,29,33,36,40,44,48,52,56,60\}$ for $4 \leqslant N \leqslant 19$. While determining $N_{c}^{\max }(N)$ for arbitrary $N$ is equivalent to the still-unsolved Erdős unit distance problem [43], it is clear that $N_{c}^{\max }(N)=3 N-6+m(N)$, where $m(N)$ grows slowly from zero to around $f(N)-(3 N-6)$ with increasing $N$.

While the maximum contact number increases (sub)linearly with $N$, the number of nonisomorphic cluster structures $|\mathcal{M}(N)|$ and transition states is assumed to increase exponentially $[11,44,45]$ [here we denote $\mathcal{M}(N)$ as the set of all nonisomorphic cluster structures of size $N$, and $|\mathcal{M}(N)|$ as the number of structures in $\mathcal{M}(N)$ ]. Stillinger showed that under certain conditions $\lim _{N \rightarrow \infty}|\mathcal{M}(N)| \propto \exp (\alpha N)$ [44]. For SHS clusters, the complete set $\mathcal{M}_{\mathrm{SHS}}\left(N, N_{c}\right)$ has been exactly determined for $N \leqslant 14$ and $3 N-6 \leqslant N_{c} \leqslant N_{c}^{\max }(N)$ via exact enumeration studies employing geometric rejection rules $[37,38]$. Unfortunately, such precise calculations are very difficult for finite-ranged potentials since exhaustive searches for energy minima are computationally intensive [46]. Only a few such studies have been performed, e.g., recent studies of $N \leqslant 19$ clusters interacting via short-range Morse potentials $[13,15,47]$.

It remains unclear how the HCR-SRA models commonly used in cluster physics relate to more physically relevant, softer interaction potentials such as the $(m, n)$-Lennard-Jones $[(m, n)$ $\mathrm{LJ}$ ] form:

$$
\left.V_{m, n}^{\mathrm{LJ}}(r)=\frac{\varepsilon}{n-m}\left[m\left(\frac{r_{e}}{r}\right)^{n}-n\left(\frac{r_{e}}{r}\right)^{m}\right] \quad \text { (with } n>m\right) .
$$

Here $\varepsilon>0$ is the dissociation energy and $r_{e}$ the equilibrium two-body interparticle distance. To simplify the presentation, we (without loss of generality) adopt reduced units $(\varepsilon=$ $1, r_{e}=1$ ) below. For $m, n \rightarrow \infty, V_{m, n}^{\mathrm{LJ}}(r) \rightarrow V_{\mathrm{SHS}}(r)$ (Fig. 1$)$, the energy landscapes of the two potentials converge in this limit. However, real systems are not in this limit. For example, for $N=13$, there are $\left|\mathcal{M}_{\text {SHS }}\right|=97,221$ stable SHS clusters $[37,38]$, but only $\left|\mathcal{M}_{\mathrm{LJ}}\right|=1,510$ stable $(m, n)=(6,12)$ LJ clusters [49]. This difference is understood qualitatively: energy landscapes are well known to support more local minima as the range of the interaction potential decreases [50,51]. There are several effects that will cause the set of stable LJ clusters to increasingly deviate from the set of stable SHS clusters as interactions become longer ranged. As $n$ and $m$ decrease, second-nearest-neighbor attractions become increasingly important, producing stable structures with $r_{i j} \leqslant 1$. Fold catastrophes [51,52] progressively eliminate stable SHS clusters, and several stable SHS structures may collapse into a single stable LJ cluster. However, detailed quantitative understanding of such effects remains rather limited.

In this paper, we quantitatively examine how stable $N \leqslant 14$ LJ cluster structures evolve away from the SHS limit as $(m, n)$ decrease. We focus on both the topography of the energy landscape [decreasing $\left|\mathcal{M}_{\mathrm{LJ}}(N)\right|$ ] and the evolving topologies of the stable cluster sets. We examine these changes in further detail for specific $N=13-14$ clusters discussed by Gregory and Newton in the 1600s in the context of the kissing number problem [40] and for a more realistic two-body potential that has been shown to accurately model rare-gas clusters [23].

\section{COMPUTATIONAL METHODS}

The pele program [53] was used to generate putatively complete sets of local minima for $(m, n)$-LJ potentials $V_{m n}^{\mathrm{LJ}}(r)$ as defined in Eq. (4). This program applies a basin-hopping algorithm that divides the potential energy surface into basins of attraction, effectively mapping each point in configuration space to a local minimum structure [54-56]. The results confirmed the number of local minima reported in previous work [57]. Finite computer time limited our search to clusters of size $N \leqslant 13$.

Starting from the SHS packings up to $N=14$, with Cartesian coordinates given by the exact enumeration algorithm [36] including rigid hypostatic clusters $\left(N_{c}<3 N-6\right)$ [38], we carried out geometry optimizations with $(m, n)$-LJ potentials using the multidimensional function minimizer from the $\mathrm{C}++$ library dlib [58]. The optimization scheme was either the Broyden-Fletcher-Goldfarb-Shanno or the conjugate gradient algorithm. The optimizations were terminated when the change in energy (in reduced units) over the course of one optimization cycle was smaller than $10^{-15}$. Subsequently, the eigenvalues of the Hessian were checked for all stationary points. If negative eigenvalues were found, the affected structures were reoptimized following displacements in both directions along the corresponding eigenvectors to locate true local minima. This procedure assures that the floppy SHS packings are successfully mapped into LJ minima.

As the optimizations often result in many duplicates, especially for small values of $n$ and $m$ where we have $\left|\mathcal{M}_{(m, n)-\mathrm{LJ}}\right| \ll\left|\mathcal{M}_{\text {SHS }}\right|$, the final structures were further analyzed and sorted. Nonisomorphic SHS clusters can be distinguished (apart from permutation of the particles) by their different adjacency matrices for $N \leqslant 13$ [38]. This is not the case for soft potentials like the LJ potential since drawing edges (bonds) between the vertices (atoms) becomes a matter of defining the distance cutoff criterion for a bond to be drawn. Therefore, we compare the interparticle distances $\left\{r_{i j}\right\}$ instead: two clusters are isomorphic (structurally identical) if they have the same ordered set of interparticle distances $\left\{r_{i j}\right\}$. While enantiomers cannot be separated using this methodology, permutation-inversion isomers are usually lumped together, since the number of distinct minima is analytically related to the order of the corresponding point group [52]. To verify the number of distinct structures, we introduced a second ordering scheme using the energy and moment of inertia tensor eigenvalues.

Two sets of structures are obtained from our optimization procedure: the first set contains all possible LJ minima $\mathcal{M}_{\mathrm{LJ}}$ from the basin-hopping algorithm, while the second set $\mathcal{M}_{\text {SHS } \rightarrow \text { LJ }}$ contains the LJ minima obtained using only the $\mathcal{M}_{\text {SHS }}$ SHS cluster structures as starting points for the geometry optimization. To compare and identify corresponding 


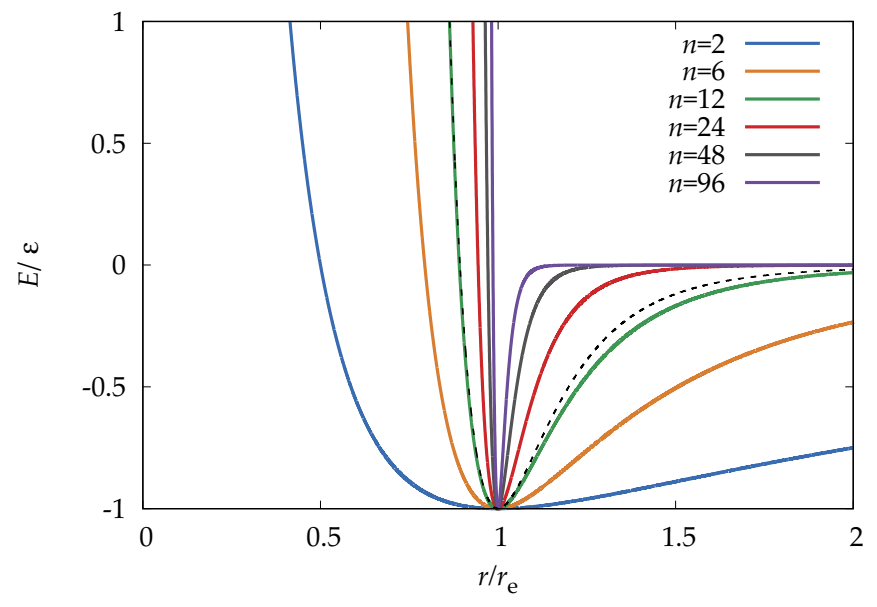

FIG. 1. Lennard-Jones potentials for different exponents $(m, n)$ with fixed $n=2 m$. As the exponents grow larger, the well of attraction becomes narrower and its shape approaches the SHS potential. The dashed line shows the extended LJ potential for the xenon dimer [48].

structures between the two sets, the $N(N-1) / 2$ interparticle distances $\left\{r_{i j}\right\}$ were again used as an identifying fingerprint.

Two-body "extended Lennard-Jones" (ELJ) potentials that accurately model two-body interactions in rare-gas clusters can be written as expansions of inverse-power-law terms [23]:

$$
V_{\mathrm{ELJ}}(r)=\sum_{n} c_{n} r^{-n},
$$

where in reduced units the condition $\sum_{n} c_{n}=-1$ holds. For comparison to the simple $(6,12)-\mathrm{LJ}$ potential, we used the ELJ potential derived from relativistic coupled-cluster theory applied to the xenon dimer, with the following coefficients for the ELJ potential (in reduced units): $c_{6}=-1.0760222355 ; c_{8}=-1.4078314494$; $c_{9}=-185.6149933139 ; \quad c_{10}=+1951.8264493941 ;$ $c_{11}=-8734.2286559729 ; \quad c_{12}=+22273.3203327203$; $c_{13}=-35826.8689874832 ; \quad c_{14}=+37676.9744744424 ;$ $c_{15}=-25859.2842295062 ; \quad c_{16}=+11157.4331408911 ;$ $c_{17}=-2745.9740079192 ; c_{18}=+293.9003309498 \quad$ [48]. The ELJ potential for xenon is shown in Fig. 1 (dashed line).

\section{RESULTS}

\section{A. Exploring the Lennard-Jones limits}

To study the convergence behavior of the number of distinct (nonisomorphic) LJ minima in the SHS limit, we performed geometry optimizations, starting from all nonisomorphic SHS structures. We will show later that the number of unique minima obtained in this procedure $\left|\mathcal{M}_{\mathrm{SHS} \rightarrow \mathrm{LJ}}\right|$ misses out only on a small portion of minima obtained from the more exhaustive basin-hopping approach: $\left|\mathcal{M}_{\mathrm{SHS} \rightarrow \mathrm{LJ}}\right| \approx\left|\mathcal{M}_{\mathrm{LJ}}\right|$. The results for a constant chosen ratio of LJ exponents $n / m=2$ are shown in Fig. 2 (top).

$\left|\mathcal{M}_{\text {SHS } \rightarrow \text { LJ }}\right|$ smoothly converges towards the SHS limit (dashed line, values in Table I) from below, thus demonstrating that for LJ systems the number of distinct minima does not grow faster than exponentially. The $(48,96)$-LJ potential has $\Delta \mathcal{M} \equiv\left|\mathcal{M}_{\mathrm{LJ}}\right|-\left|\mathcal{M}_{\mathrm{SHS} \rightarrow \mathrm{LJ}}\right|=$
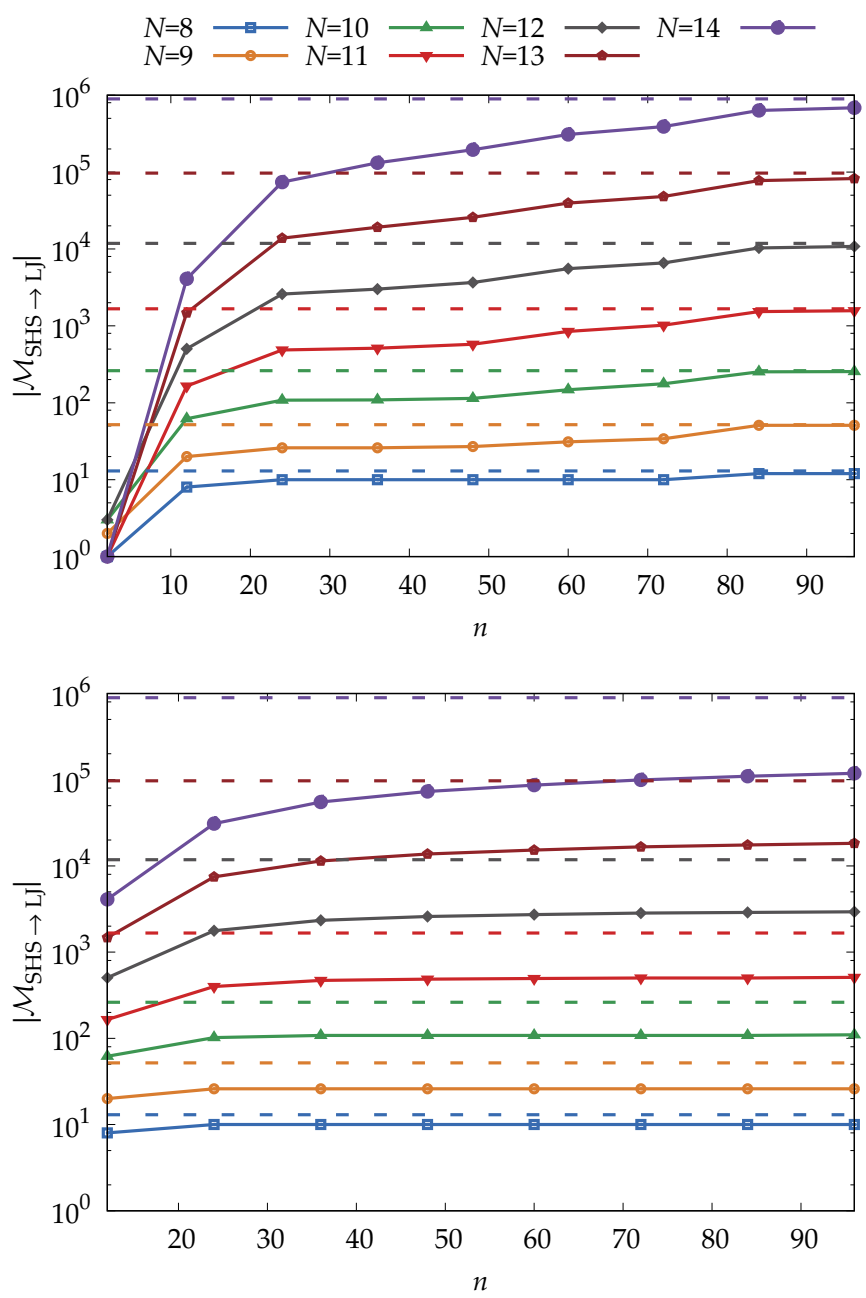

FIG. 2. Convergence of the number of distinct LJ local minima $\left|\mathcal{M}_{\text {SHS } \rightarrow \text { LJ }}\right|$ obtained through geometry optimizations starting from the nonisomorphic SHS structures with increasing LJ exponent $n$. Permutation-inversion isomers and enantiomers are not distinguished. The dashed line gives the exact SHS limit $\left|\mathcal{M}_{\text {SHS }}\right|$. Top panel: $m=$ $n / 2$. Bottom panel: fixed $m=6$.

$\{1,1,7,91,1019,14507,207697\}$ fewer stable minima than the SHS potential. The fractions of missing minima $\Delta \mathcal{M} /\left|\mathcal{M}_{\text {SHS }}\right|$ for this potential grow with increasing $N$ and are, respectively, $\{7.69,1.92,2.67,5.46,8.62,14.92,23.19\} \%$. Note that for $N \geqslant 10$ most of these missing minima correspond to highenergy $\left(N_{c}<N_{c}^{\max }\right)$ structures.

If the exponent $n$ for the repulsive part of the LJ potential is increased with $m$ kept constant, the LJ potential becomes equivalent to the SHS potential in the repulsive range but remains attractive at long range. Figure 2 (bottom) shows the convergence of the number of unique structures with respect to $n$ at set $m=6$ towards the SHS limit. Here the number of distinct minima converges towards a number that is much smaller than the total number of SHS packings, demonstrating that (as expected) the attractive part of the potential contributes significantly to the decrease of the number of local minima compared to the rigid SHS model.

To see if the asymptotic increase in the number of distinct minima $|\mathcal{M}(N)| \sim e^{\alpha N}$ is indeed exponential, we use 
TABLE I. Number of distinct local minima $\left|\mathcal{M}_{\mathrm{SHS}}\right|$ for cluster size $N$ (from Refs. [36-38]) and contact number $N_{c}$ from the exact enumeration, compared to the number of different structures obtained from a geometry optimization starting from the set $\mathcal{M}_{\mathrm{SHS} \rightarrow \mathrm{LJ}}\left(N, N_{c}\right)$ for a $(6,12)-\mathrm{LJ}$ potential. The overall number of unique minima $\left|\mathcal{M}_{\mathrm{SHS} \rightarrow \mathrm{LJ}}\right|=\sum_{N_{c}}\left|\mathcal{M}_{\mathrm{SHS} \rightarrow \mathrm{LJ}}\left(N_{c}\right)\right|-$ (no. of duplicate structures) is shown in the following column. This result can be compared to the number of unique minima found using the basin-hopping method $\left(\left|\mathcal{M}_{\mathrm{LJ}}\right|\right)$. The difference $\Delta \mathcal{M}=\left|\mathcal{M}_{\mathrm{LJ}}\right|-\left|\mathcal{M}_{\mathrm{SHS} \rightarrow \mathrm{LJ}}\right|$ is also listed.

\begin{tabular}{|c|c|c|c|c|c|c|}
\hline$N$ & $N_{c}$ & $\left|\mathcal{M}_{\mathrm{SHS}}\left(N_{c}\right)\right|$ & $\left|\mathcal{M}_{\mathrm{SHS} \rightarrow \mathrm{LJ}}\left(N_{c}\right)\right|$ & $\left|\mathcal{M}_{\mathrm{SHS} \rightarrow \mathrm{LJ}}\right|$ & $\left|\mathcal{M}_{\mathrm{LJ}}\right|$ & $\Delta \mathcal{M}$ \\
\hline 8 & 18 & 13 & 8 & 8 & 8 & 0 \\
\hline 9 & 21 & 52 & 20 & 20 & 21 & 1 \\
\hline \multirow[t]{3}{*}{10} & 23 & 1 & 1 & \multirow{3}{*}{62} & \multirow{3}{*}{64} & \multirow{3}{*}{2} \\
\hline & 24 & 259 & 60 & & & \\
\hline & 25 & 3 & 3 & & & \\
\hline \multirow[t]{5}{*}{11} & 25 & 2 & 2 & \multirow{5}{*}{165} & \multirow{5}{*}{170} & \multirow{5}{*}{5} \\
\hline & 26 & 18 & 6 & & & \\
\hline & 27 & $1620^{\mathrm{a}}$ & 158 & & & \\
\hline & 28 & 20 & 12 & & & \\
\hline & 29 & 1 & 1 & & & \\
\hline \multirow[t]{6}{*}{12} & 28 & 11 & 6 & \multirow{6}{*}{504} & \multirow{6}{*}{515} & \multirow{6}{*}{11} \\
\hline & 29 & 148 & 24 & & & \\
\hline & 30 & 11638 & 483 & & & \\
\hline & 31 & 174 & 69 & & & \\
\hline & 32 & 8 & 6 & & & \\
\hline & 33 & 1 & 1 & & & \\
\hline \multirow[t]{6}{*}{13} & 31 & 87 & 23 & \multirow{6}{*}{1476} & \multirow{6}{*}{1510} & \multirow{6}{*}{34} \\
\hline & 32 & 1221 & 100 & & & \\
\hline & 33 & $95810^{\mathrm{a}}$ & 1418 & & & \\
\hline & 34 & $1318^{a}$ & 293 & & & \\
\hline & 35 & 96 & 49 & & & \\
\hline & 36 & 8 & 6 & & & \\
\hline \multirow[t]{8}{*}{14} & 33 & 1 & 1 & \multirow{8}{*}{4093} & \multirow{8}{*}{$(4187)^{b}$} & \multirow{8}{*}{$(94)^{b}$} \\
\hline & 34 & 707 & 101 & & & \\
\hline & 35 & 10537 & 410 & & & \\
\hline & 36 & 872992 & 3939 & & & \\
\hline & 37 & 10280 & 1002 & & & \\
\hline & 38 & 878 & 237 & & & \\
\hline & 39 & 79 & 42 & & & \\
\hline & 40 & 4 & 3 & & & \\
\hline
\end{tabular}

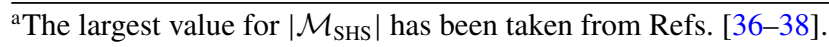

${ }^{\mathrm{b}}$ Estimated.

Stillinger's expression for the asymptotic exponential rise rate parameter is [44],

$$
\alpha=\lim _{N \rightarrow \infty}\left[N^{-1} \ln |\mathcal{M}(N)|\right]
$$

Figure 3 shows the number of distinct minima for SHS clusters obtained from the data shown in Table I. The $N \geqslant 12$ SHS data give $\alpha_{\text {SHS }} \approx 2.21$. Figure 3 also shows the $(6,12)$-LJ results obtained using basin-hopping; these yield $\alpha_{\mathrm{LJ}} \approx 1.10$, which is close to the $\alpha=0.8$ value estimated by Wallace [59] or to the recently given value of 1.04 by Forman and Cameron [45]. Note that the rapid increase of $\left|\mathcal{M}_{\mathrm{SHS}}\right| /\left|\mathcal{M}_{\mathrm{LJ}}\right|$ with $N$ is explained by the much larger values of $\alpha$ for the SHS compared to the LJ clusters.

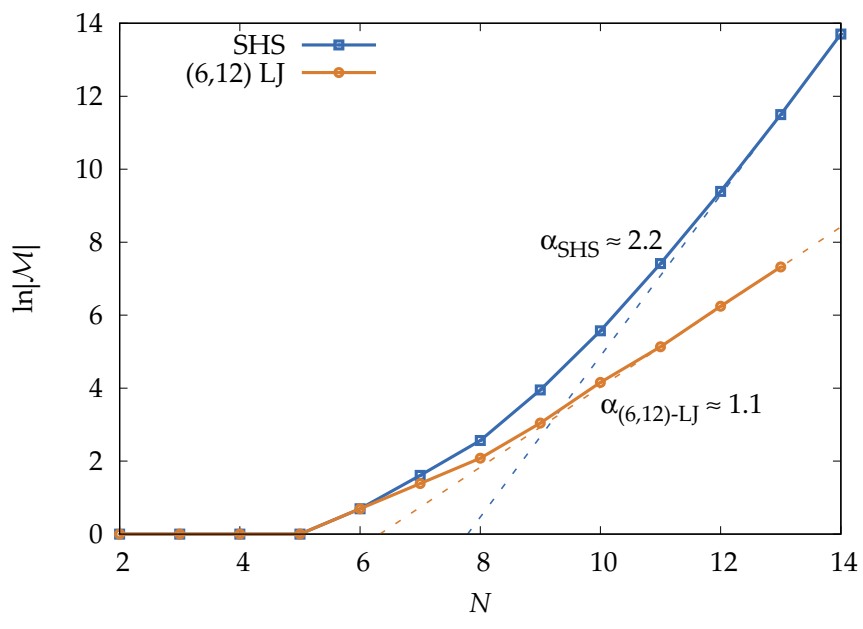

FIG. 3. Growth behavior of $|\mathcal{M}(N)|$ of SHS and $(6,12)$-LJ clusters and corresponding asymptotic exponential rise rate parameter $\alpha$ for $N \geqslant 12$ as defined in Eq. (6). The intercepts $\ln |\mathcal{M}(N=0)|$ are -17.19 and -6.94 for the SHS and $(6,12)$-LJ cases, respectively.

Using the results for $N \geqslant 13$ from Fig. 2, we can calculate how $\alpha$ depends on the LJ range parameter $n$. As shown in Fig. 4, a general function of the form

$$
\alpha(n)=\alpha_{\max }+\frac{a}{\left(n-n_{0}\right)^{p}}
$$

fits the results nicely, allowing the prediction of growth behavior for different LJ potentials. For $\left|\mathcal{M}_{(n / 2, n)-\mathrm{LJ}}\right|, \alpha_{\max }$ is equivalent to $\alpha_{\mathrm{SHS}}=2.207$. The other adjusted parameters are $a=-66.588, n_{0}=-3.386$, and $p=1.473$ (Fig. 4). We also show the ratio $\alpha\left(\left|\mathcal{M}_{\mathrm{SHS} \rightarrow(n / 2, n)-\mathrm{LJ}}\right|\right) / \alpha\left(\left|\mathcal{M}_{\mathrm{SHS} \rightarrow(6, n)-\mathrm{LJ}}\right|\right)$ between the two different LJ asymptotic exponential rise rate parameters, which shows that larger cluster sizes need to be studied to correctly describe the asymptotic limit.

The distribution of minima as a function of (free) energy was suggested to be Gaussian [60]. Figure 5 shows the energy

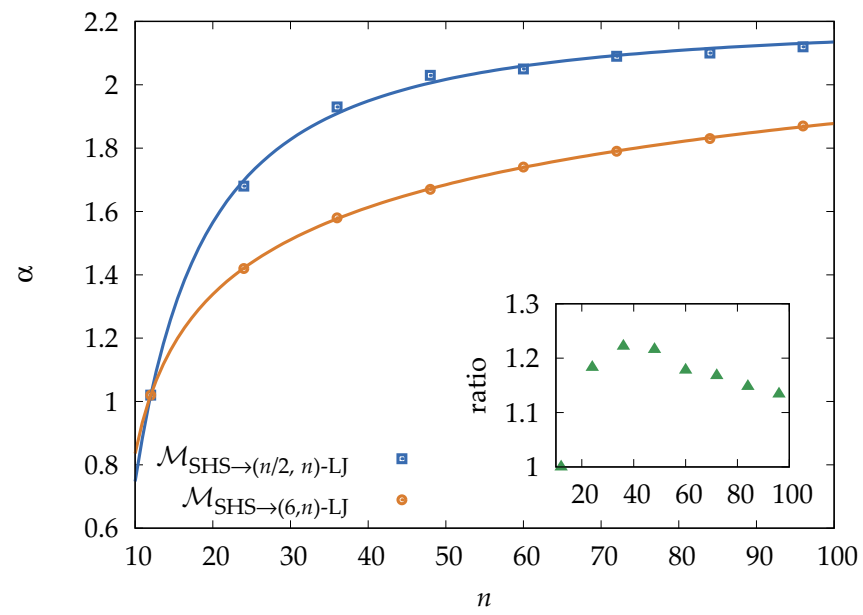

FIG. 4. Convergence behavior of the asymptotic exponential rise rate parameter $\alpha$ [Eq. (6)] towards the SHS limit with respect to the LJ exponent $n$. The inset shows the ratio of the two quantities $\alpha\left(\left|\mathcal{M}_{\mathrm{SHS} \rightarrow(n / 2, n)-\mathrm{LJ}}(N)\right|\right) / \alpha\left(\left|\mathcal{M}_{\mathrm{SHS} \rightarrow(6, n)-\mathrm{LJ}}(N)\right|\right)$. 


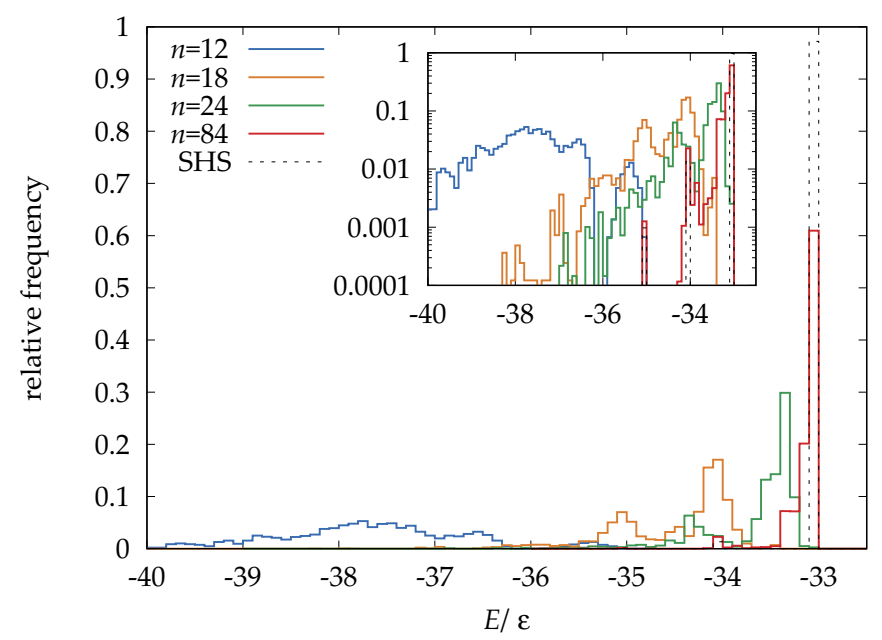

FIG. 5. Histogram of the energies (bin size $\Delta \varepsilon=0.1$ ) of minima $\mathcal{M}_{\mathrm{SHS} \rightarrow(n / 2, n)-\mathrm{LJ}}(N)$ for $N=13$ and different exponents $n$ up to the SHS limit. For better visibility, the height of the bars is set to $\Delta|\mathcal{M}| /|\mathcal{M}|$ in the interval $\Delta(E / \epsilon)$. The inset shows the same data in logarithmic scale.

distribution of minima for different $\mathrm{LJ}(n / 2, n)$ potentials derived from SHS initial structures. We do not see a Gaussian type of distribution; this result does not change if we take the free energy at finite temperatures. The results indicate a "phase transition" in the potential energy landscape away from low-energy to high-energy minima as $n$ increases. The transition occurs at fairly small $n$. Results for the $(9,18)-\mathrm{LJ}$ potential indicate two HCR-SCA-like maxima that are not present for the $(6,12)$-LJ potential; these are associated with the $N_{c}=34$ and $N_{c}=35$ SHS clusters, respectively. It is also clear that (as expected) the distributions narrow with increasing $n$.

It is well known that the global energy minimum for rare gas clusters with 13 atoms is the ideal Mackay icosahedron [61-63]. Simple geometric considerations imply that such a symmetric cluster is not possible for sticky hard spheres; all vertices of a regular icosahedron with unit edge length lie on a circumscribing sphere with radius $r_{c} \approx 0.951$, making it impossible to insert a sphere of the same radius into the center of the polyhedron. Therefore, there must be well-defined LJ exponents $(m, n)$ at which the icosahedral $N=13 \mathrm{LJ}$ cluster breaks symmetry to form a rigid cluster. For the $n=2 m$ case considered above, this symmetry breaking occurs at $m \simeq 15$.

We also explored a more realistic extended LJ potential [Eq. (5); Fig. 1] for one of the rare gas dimers (xenon) in comparison with other LJ potentials. We see that the repulsive part agrees nicely with the conventional $(6,12)$ LJ potential, while for $r>1$ the extended LJ potential is slightly less attractive. This change should lead to an increase in the number of local minima compared to the conventional $(6,12)-\mathrm{LJ}$ potential. We find that this is indeed the case, i.e., $\left|\mathcal{M}_{\mathrm{SHS} \rightarrow \mathrm{ELJ}}\right|=\{8,21,74,205,685,2179,6863\}$ for $N=\{8,9,10,11,12,13,14\}$. For $N=13$ the number of distinct minima is $44 \%$ larger than it is for the simple $(6,12)-\mathrm{LJ}$ potential, which shows that $|\mathcal{M}(N)|$ is rather sensitive to the potential chosen. Hence, to correctly describe the topology of real systems, one has to take care of the correct form of the two-body contribution (as well as higher $n$-body contributions) [25].

\section{B. $(6,12)-L J$ clusters from basin-hopping}

Table I shows the number of distinct minima found by our cluster geometry optimization procedure using the $(6,12)-\mathrm{LJ}$ potential compared to results from exact enumeration for SHSs and from basin-hopping for the $(6,12)$-LJ potential. As the SHS clusters for a specific $N$ value can be grouped by their contact number $N_{c}$, the geometry optimizations were carried out separately for each group of $\mathcal{M}_{\text {SHS }}\left(N_{c}\right)$. Hoy $[36,37]$ and Holmes-Cerfon [38] reported slightly different results for $N=11$ and $N=13$; we find that upon geometry optimization, their datasets yield the same final clusters $\left|\mathcal{M}_{\mathrm{SHS} \rightarrow \mathrm{LJ}}\left(N_{c}\right)\right|$. As identical LJ clusters appear in multiple groups with different contact numbers, we remove the duplicates to create the set $\mathcal{M}_{\text {SHS } \rightarrow \text { LJ }}$ of distinct minima, which can be directly compared to the set of $\mathrm{LJ}$ minima $\mathcal{M}_{\mathrm{LJ}}$ obtained from the basin-hopping method. It should be noted that including the hypostatic clusters and the different $\left|\mathcal{M}_{\mathrm{SHS}}\right|$ for $N=11$ and $N=13$ from Ref. [38] did not change our results, implying that hypostatic clusters are not an important feature for the LJ energy landscape.

Interestingly, our gradient-based minimization procedure starting from the SHS packings does not in general lead to a complete set of LJ minima; the mapping from SHS minima to LJ minima is noninjective and nonsurjective. Clearly, some structural motifs found in LJ clusters are not found in SHS

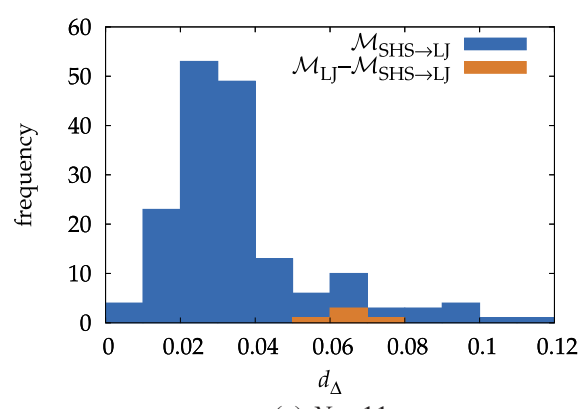

(a) $N=11$

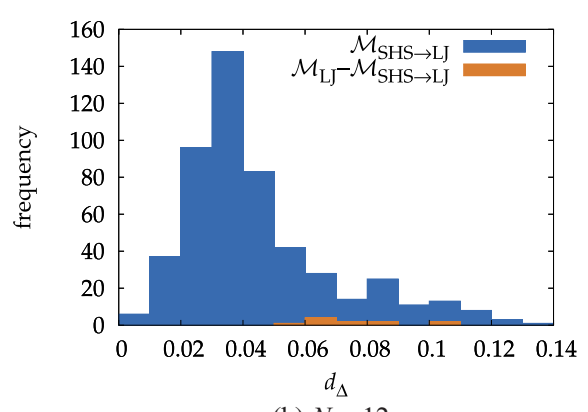

(b) $N=12$

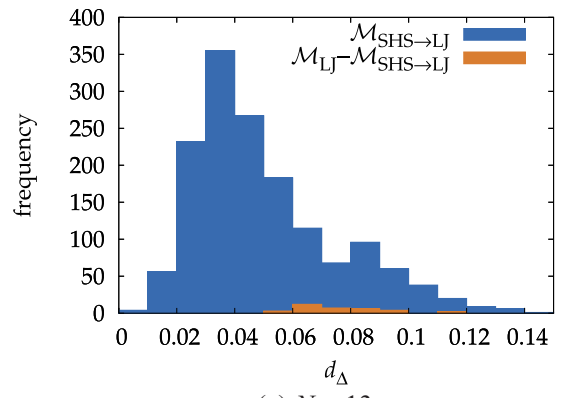

(c) $N=13$

FIG. 6. Histograms of the difference between the longest and shortest bond distances $d_{\Delta}=d_{\max }-d_{\min }$ for the complete set of distinct LJ minima $\mathcal{M}_{\mathrm{LJ}}(N)$ for $N=\{11,12,13\}$. Orange bars give the number of distinct structures not contained in $\mathcal{M}_{\mathrm{LJ}}$ as obtained from the basin-hopping algorithm. 
TABLE II. Range $\left[E_{0}, E_{\max }\right]$ of the energy spectrum of all $\mathrm{LJ}$ minima, position of the second lowest minimum structure $E_{1}$, and position of the first unmatched (UM) structure $E_{0}^{\mathrm{UM}}$ relative to the respective global minimum (in reduced units and $E_{0}=0$ ).

\begin{tabular}{lccc}
\hline \hline$N$ & $E_{\max }$ & $E_{1}$ & $E_{0}^{\mathrm{UM}}$ \\
\hline 8 & 1.04 & 0.06 & - \\
9 & 2.08 & 0.84 & 1.19 \\
10 & 3.13 & 0.87 & 2.22 \\
11 & 4.22 & 0.85 & 2.27 \\
12 & 6.16 & 1.62 & 3.38 \\
13 & 9.26 & 2.85 & 6.14 \\
\hline \hline
\end{tabular}

clusters and vice versa, and the topology of the hypersurface changes in a nontrivial fashion from SHS to LJ. However, it is surprising that the fraction of structures that are missed by this optimization procedure is so small (see Table III). To gain further insight, we analyzed the energetics and structure of the unmatched clusters in more detail.

Figure 6 shows an analysis of the difference between the longest to the shortest bond lengths $d_{\Delta}=d_{\max }-d_{\min }$ obtained for the largest clusters in $\mathcal{M}_{\mathrm{LJ}}$ with $N=\{11,12,13\}$ [64]. The histograms show that the clusters most commonly have a $d_{\Delta}$ of about 0.03 . In contrast, as shown by the orange bars, the unmatched structures have significantly larger $d_{\Delta}$ values of at least 0.05 , with most of them having $d_{\Delta} \simeq 0.06$. This is a first indication of why these structures are not found by starting from SHS packings. The latter form bonds only of length one, and a large variation in bond length could imply that a SHS packing similar to the LJ structure does not exist as the SHS geometrical conditions are not satisfied. The data in Table II show that the unmatched (UM) structures for a specific $N$ value have much higher energies compared to the one of the global minimum (which is set to zero, i.e., $E_{0}=0$ ). They are always positioned in the upper half of the energy spectrum, making them energetically unfavorable. However, we could not find any correlation between $d_{\Delta}$ and the energetic position of the LJ clusters.

Last, we checked the geometries of the missing structures in more detail. As it turns out, almost all of the missing stable LJ clusters can be created from a smaller set of missing clusters by capping some of their triangular faces. Therefore, these groups of clusters can be referred to as "seeds" [35]. The corresponding starting structures of each seed are shown in Fig. 7. None of these structures are stable SHS packings. For example, structure (d) can be described as three octahedra connected via triangular faces sharing one edge. Geometric considerations $[35,36]$ immediately show that this structure cannot be a stable SHS packing; the dihedral angle in an octahedron is approximately $109.5^{\circ}$, which means three octahedra fill only $328.5^{\circ}$ of a full circle, leaving a gap between two faces.

Table III shows the number of missing minima belonging to each seed. More than $60 \%$ of the unmatched structures belong to seeds (a) and (b). From a graph theoretical point of view [34,35], grouping structures into seeds means that all structures belonging to the same seed contain the graph of the starting structures as a subgraph in their respective connectivity matrix. This approach simplifies the analysis to a great extent,

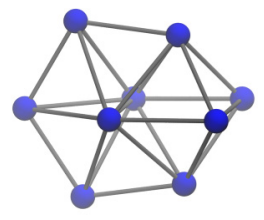

(a)

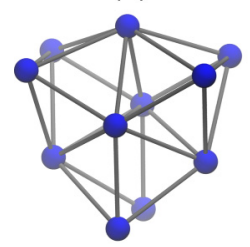

(d)

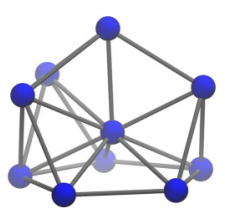

(b)

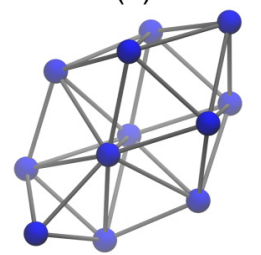

(e)

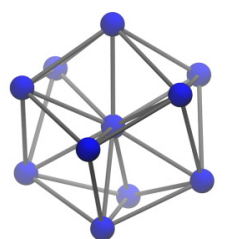

(c)

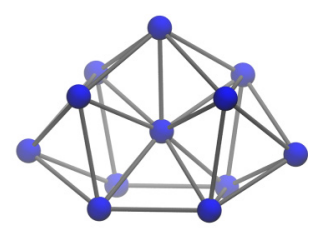

(f)
FIG. 7. Graphical representations of the structures that are starting new seeds, but are not contained in $\mathcal{M}_{\mathrm{SHS} \rightarrow \mathrm{LJ}}$. See Table III and text for more details.

as the feature that prevents the structures from being found by geometry optimization is the same for each of the structures arising from a specific seed. The smallest unmatched structures that cannot be associated with any of seeds (a)-(f) have $N=13$; these could be the starting structures for two new seeds.

Finally, we note that the starting SHS minima in our optimization procedure are not stationary points on the LJ hypersurface, and we therefore optimize to most but not all local and available LJ minima. This observation explains why some high-energy structures were not found by our optimization procedure. For a smooth change in the topology of the potential energy surface from SHS to LJ type clusters one has to continuously vary the exponents $(m, n)$ in real space, which is computationally too demanding.

TABLE III. Number of missing structures after optimization belonging to the same "seed" (Fig. 7). $N=8$ is excluded because all LJ minima were found starting from the SHS model.

\begin{tabular}{lccccc}
\hline \hline Seed & $N=9$ & $N=10$ & $N=11$ & $N=12$ & $N=13$ \\
\hline $\mathrm{a}$ & 1 & 1 & - & 3 & 8 \\
$\mathrm{~b}$ & - & 1 & 3 & 4 & $12^{\mathrm{a}}$ \\
$\mathrm{c}$ & - & - & 1 & $1^{\mathrm{a}}$ & - \\
$\mathrm{d}$ & - & - & 1 & 1 & 5 \\
$\mathrm{e}$ & - & - & - & 1 & 6 \\
$\mathrm{f}$ & - & - & - & 1 & 1 \\
Remaining & - & - & - & - & 2 \\
Total & 1 & 2 & 5 & 11 & 34 \\
$\%$ & 4.76 & 3.13 & 2.94 & 2.14 & 2.25 \\
\hline \hline
\end{tabular}

a Some structures undergo a slight rearrangement from a perfect capped structure. Specifically, two structures belonging to seed (b) and one structure belonging to seed (c) were found to deviate slightly from the perfect arrangement, but minor rearrangements of these structures lead to the desired geometry, and they can be reasonably associated with these seeds. 


\section{The special case of a Gregory-Newton cluster}

We call a cluster "Gregory-Newton" (GN) when it belongs to the set of all clusters consisting of 12 spheres kissing a central sphere. The canonical GN cluster is the icosahedron, which is perhaps the most common form studied in cluster chemistry and physics $[2,41,65,66]$. We therefore investigate this cluster type in more detail here.

For monodisperse SHS clusters, the GN argument (as proved by Schütte and van der Waerden [40]) that no more than 12 equally sized spheres can touch a central sphere of the same size holds. We note that the problem of the number of kissing spheres in $k$ dimensions, or even in three dimensions with sphere size smaller than that of the central sphere, remains largely unsolved [41]. For unequally sized spheres, some simple results related to spherical codes [67] are known; for example, 13 hard spheres of radius $r_{s}$ can touch a central sphere of unit radius only if $r_{s} \leqslant 0.9165$ [67]. For particles interacting via finite-ranged potentials such as $V_{m n}^{\mathrm{LJ}}(r)$, however, the situation is far more complicated since systems minimize energy rather than differences in the distances between neighboring particles, and few general results are known. Nonetheless, this latter problem is important for understanding real systems such as coordination compounds [68], which have recently been shown to possess coordination numbers as high as 17 [69] or even 20 [70].

Motivated by these recent results, we investigated longer range potentials by decreasing the $\mathrm{LJ}$ exponents $(m, n)$, to see whether the restriction of no more than 12 kissing equal-sized spheres still holds. As it is impossible to distribute 13 points on a sphere evenly (there is no triangulation of a sphere with 13 vertices of degree 5 and 6 [71]), we used the Fibonacci sphere algorithm [72,73] to find an approximate distribution of points on a sphere and added a center sphere. By optimizing the coordinates for this $N=14$ cluster with different $L J$ exponents and calculating the distance of every sphere to the center sphere, we can deduce at which "softness" a 13th sphere is (perhaps) allowed to enter the first coordination shell, i.e., to touch the center sphere.

Figure 8 shows the difference between the largest and the smallest center-to-outer sphere (COS) distances in relation to the $\mathrm{LJ}$ exponents $m$ and $n$. Interestingly, none of the $(m, n)$-LJ potentials lead to equal distances around a central sphere. While this result could be due to the lack of symmetry, one sphere is clearly farther away from the central sphere even for the softest "Kratzer" $(1,2)-\mathrm{LJ}$ potential [74]. For this potential the largest and smallest COS distances are $r_{\max }=0.882$ and $r_{\min }=0.804$, respectively. While the longest distance shows up only once, the shortest distance appears twice. All other 10 distances fall in the range between $r=0.845$ and $r=0.861$. The $r_{\max } / r_{\min }$ ratio is 1.097 and is much smaller than $r_{\max } / r_{\min }=\sqrt{2}$ for the closed packed lattice, or the shortest distance possible for the SHS system, which is $r_{14}^{\mathrm{GN}}=1.347$ (see discussion below). Hence the 13th sphere "almost" touches the center sphere.

Note that all COS distances for the $N=14(1,2)$-LJ cluster are significantly shorter than $r=1$, due to the $N(N-1) / 2$ attractive two-body interactions and the softness of the potential. For infinite (e.g., body-centered cubic or close-packed) lattices of particles interacting via $V_{m n}^{\mathrm{LJ}}(r)$ with $n>m>3$,

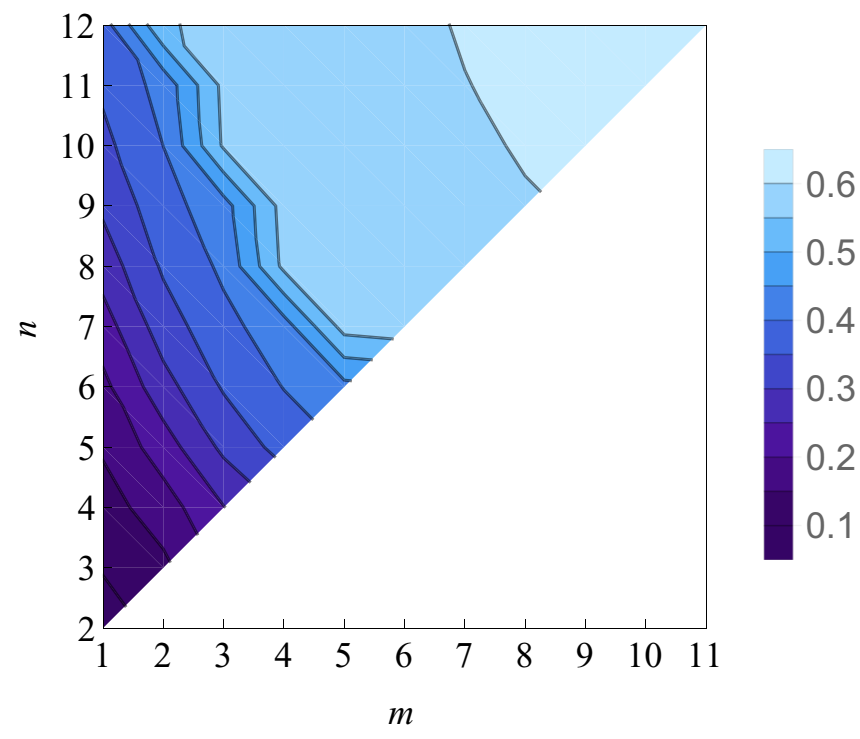

FIG. 8. Relation of LJ exponents $m$ and $n$ to the difference of largest and smallest COS distances. A value of zero would imply that all surrounding spheres are touching the center sphere.

one can prove [23] that the nearest-neighbor distance is

$$
r_{\mathrm{NN}}(m, n)=\left(L_{n} L_{m}^{-1}\right)^{\frac{1}{n-m}} .
$$

Here $L_{n}$ is the Lennard-Jones-Ingham lattice coefficient for a specific lattice determined from 3D lattice sums. Since $L_{n}<L_{m}$ for $n>m$, we see that $r_{\mathrm{NN}}<1$, and $\quad \lim _{m, n \rightarrow \infty} r_{\mathrm{NN}}(m, n)=1$. The shortest distances found in $(6,12)-\mathrm{LJ}$ clusters $r_{\min }(N)$ are $\quad r_{\min }(8)=0.986767, r_{\min }(9)=0.964404, r_{\min }(10)=$ $0.964382, r_{\min }(11)=0.956345, \quad r_{\min }(12)=0.947842, \quad$ and $r_{\min }(13)=0.952179$. Surprisingly, $r_{\min }(12)$ is smaller than $r_{\mathrm{NN}}(6,12)$ for typical crystalline lattices; $r_{\mathrm{NN}}(6,12)$ values are $0.95066,0.95186$, and 0.97123 for simple cubic, body-centered cubic, and close-packed lattices, respectively. This result shows that stable clusters do not necessarily have longer bonds compared to the solid state, where we expect a maximum in interaction energy per atom.

Finally, we relate the above results back to the motifs present in the HCR-SRA limit by focusing on $N=13$ and $N=14$ SHS clusters from Ref. [38]. This set contains all nonisomorphic SHS structures that can be considered GN clusters $(N=13)$ and the $N=14$ structures that can be derived from them by attaching a 14th sphere. We find a surprisingly large number (737) of nonisomorphic $N=13$ GN-SHS structures $\left(\{724,10,1,2\}\right.$ for $\left.N_{c}=\{33,34,35,36\}\right)$, that all optimize to the ideal icosahedral arrangement $\left(I_{h}\right.$ symmetry) if a $(6,12)$-LJ potential is applied. An even larger number of clusters exists for $N=14$ (14 529), which is $\approx 0.016\left|\mathcal{M}_{\mathrm{SHS}}(14)\right|$. All of these structures optimize to just one of two possible $(6,12)$-LJ minima of GN type. The first is the Mackay icosahedron capped on one of its triangular faces, and the second is an elongated pentagonal bipyramid (belonging to the class of Johnson solids) with the 14th sphere capping a square face. 

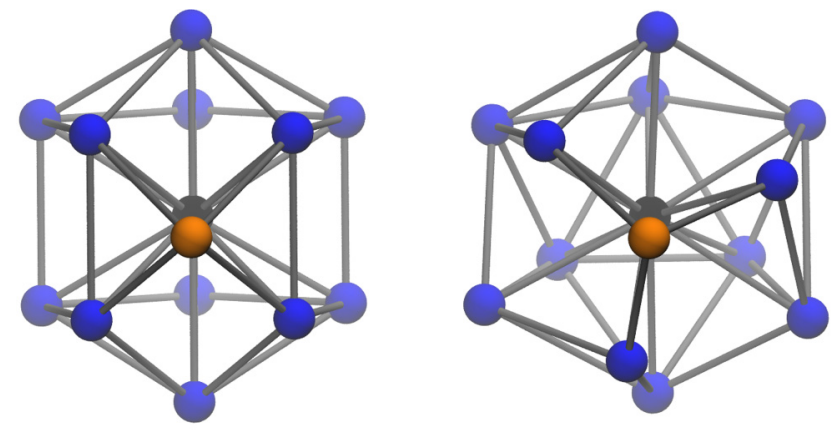

(a) $r_{14}^{\mathrm{GN}}=1.34715, N_{c}=39$
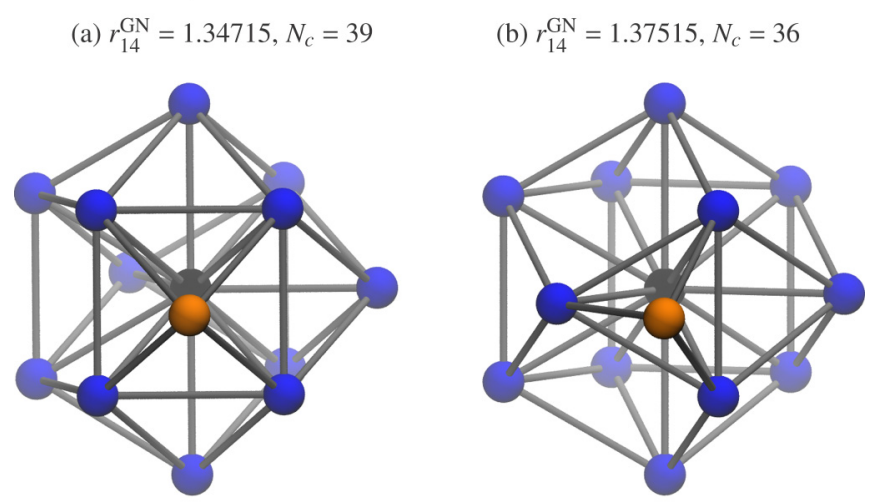

(c) $r_{14}^{\mathrm{GN}}=\sqrt{2}, N_{c}=40$

FIG. 9. Graphical representations of SHS packings with $N=14$, where a center sphere is maximally contacting. The orange sphere in each cluster is the 14th outer sphere, not able to touch the center sphere (in black): (a) distorted elongated pentagonal bipyramid (Johnson solid), (b) distorted icosahedron, (c) hcp capped on a square, (d) hcp capped on a triangle.

Most of these $N=14$ clusters are minimally rigid $\left(N_{c}=3 N-6=36\right)$, while only a few are hyperstatic $\left(N_{c}>3 N-6\right)$ and none are hypostatic $\left(N_{c}<3 N-6\right)$. There are $\{14369,144,8,6,2\}$ such clusters with $N_{c}=$ $\{36,37,38,39,40\}$ and $N=14$. The clusters with $N_{c}=40$ are hcp and fcc core-shell structures capped at a square face; these arrangements maximize $N_{c}$. Most of the clusters with $N_{c}=\{38,39\}$ are deformed versions of the elongated pentagonal bipyramid mentioned above, indicating that this arrangement is a favored route to these intermediate-energy structures. However, $N_{c}=39$ also contains hcp and fcc structures capped at a triangular face. The first example of a cluster derived from a perfect icosahedral symmetry shows up at lower value $N_{c}=37$. Representative examples for clusters with high contact numbers are depicted in Fig. 9.

Surprisingly, the $N=14$ cluster with the closest COS distance $r_{\min }^{\mathrm{COS}}$ was not known. Here we close this gap by determining the $\mathrm{COS}$ distance for all $\mathrm{GN}$-type clusters. We find one single cluster with $r_{\mathrm{min}}^{\mathrm{COS}}=1.3471506281091$. Its structure [Fig. 9(a)] is similar to the elongated pentagonal bipyramid (a Johnson solid) with one of the square faces stretched to form a regular rectangle. The 14th sphere caps this deformed face, becoming the vertex of a deformed octahedron and allowing the outer sphere to get closer to the central sphere. The next-smallest- $r{ }^{\mathrm{COS}}$ cluster $\left(r^{\mathrm{COS}}=1.37515\right)$ is shown in

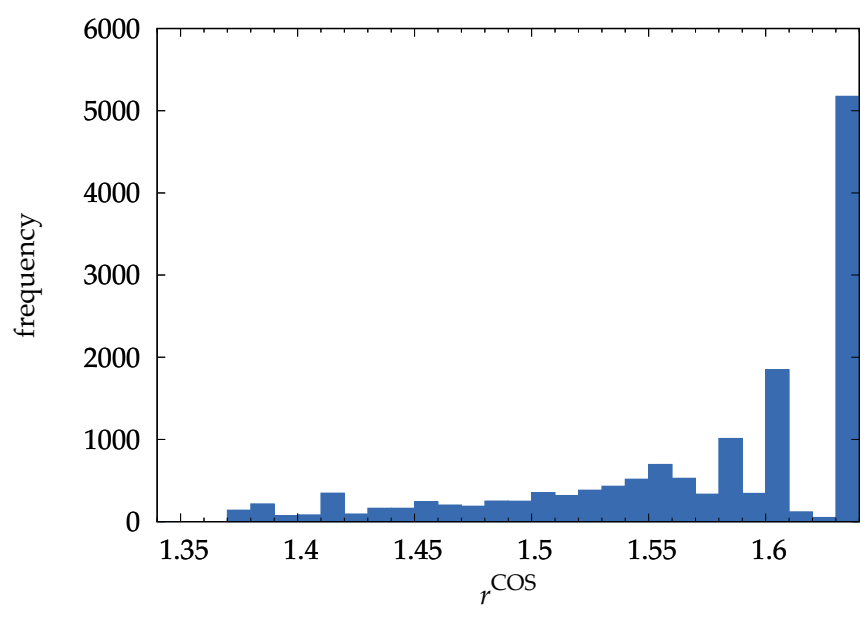

FIG. 10. Frequency of distances from the cluster center to the most distant sphere for all GN-like clusters contained in the structures from Ref. [38]. The width of the bars is 0.01 .

Fig. 9(b). It does not belong to the category of the clusters derived from the elongated pentagonal bipyramid, but instead can be described as being icosahedral-like. The short distance is achieved by attaching the 14th sphere to three spheres that do not form a face of the cluster (because they are separated by a distance larger than 1 ).

As shown in Fig. 10, the distribution of $r^{\mathrm{COS}}$ values for the full set of GN clusters is shown in Fig. 10. Motifs with larger $r^{\mathrm{COS}}$ are far more prevalent. For example, the peak at $r^{\mathrm{COS}}=1.41$ corresponds to structures where the 14 th sphere is touching four other spheres that are part of a tetragonal pyramid, therefore forming a regular octahedron with a tipto-tip distance of $\sqrt{2}$ [Fig. 9(c)]. The maximum $r^{\mathrm{COS}}$ value (1.63) corresponds to capping triangular faces, so that the most distant sphere is part of a regular trigonal bipyramid with a height of $\sqrt{8 / 3}$ [Fig. 9(d)]. The structures corresponding to the bars at $1.60,1.58$, and 1.55 are derived from the regular trigonal bipyramid and result from breaking its axial bonds. In these structures, the more bonds are broken, or the further the axial spheres are separated, the shorter the COS distance becomes.

\section{CONCLUSIONS}

We have characterized the sets of $(m, n)$-LJ-potential minima obtained using complete sets of nonisomorphic SHS packings with $8 \leqslant N \leqslant 14$ [34-38] as initial states for energy minimization. The number of distinct minima (i.e., excluding permutation-inversion isomers) is far smaller than the number of SHS packings for the standard Lennard-Jones exponents $(m, n)=(6,12)$, but approaches the SHS limit from below as $(m, n)$ increase. We characterized how the number of distinct minima $\mathcal{M}(N)$ increases with cluster size $N$ by determining Stillinger's rise rate parameter $\alpha$ [Eq. (6) [44]]. The increase of $\alpha$ from $\approx 1.1$ for $(6,12)$-LJ clusters to $\approx 2.2$ for SHS clusters is described by a simple functional form [Eq. (7)]. All these results can be understood in terms of a smooth progression of the $(m, n)$-LJ energy landscape towards the SHS energy landscape as $(m, n)$ increase. 
Using a more realistic extended LJ potential obtained from coupled cluster calculations for the xenon dimer $[23,48]$ leads to $\mathcal{M}$ values close to those obtained for the $(6,12)-\mathrm{LJ}$ potential, but our results indicate the the topology of the energy hypersurface is very sensitive to the model potential applied. For softer potentials, we showed that it is still unfavorable for a 13th outer sphere to touch the center sphere. Indeed, the Gregory-Newton argument [75] still holds true for even the softest $(m, n)=(1,2)$ potential.

Finally, we compared our optimization results to the previously published results for the $(6,12)$-LJ potential. The mapping from $\mathcal{M}_{\mathrm{SHS}}$ to $\mathcal{M}_{\mathrm{SHS} \rightarrow \mathrm{LJ}}$ is noninjective and nonsurjective; however, the number of structures missed by the optimization procedure is relatively small. The unmatched structures belong to the high-energy region of the potential energy hypersurface and possess rather large variations in their bond lengths. An analysis of their geometries revealed that most of the larger structures can be constructed from a smaller cluster by capping some of the triangular faces. This procedure effectively sorts almost all unmatched structures into six seeds for clusters up to $N=13$.

\section{ACKNOWLEDGMENTS}

We acknowledge financial support by the Marsden Fund of the Royal Society of New Zealand (MAU1409). D.J.W. gratefully acknowledges financial support from the EPSRC. P.S. acknowledges financial support by the Centre for Advanced Study at the Norwegian Academy of Science and Letters (Molecules in Extreme Environments Research Program). We thank Dr. Lukas Wirz and Dr. Elke Pahl for useful discussions.
[1] F. H. Stillinger and T. A. Weber, Science 225, 983 (1984).

[2] T. Martin, Phys. Rep. 273, 199 (1996).

[3] J. P. K. Doye and D. J. Wales, Science 271, 484 (1996).

[4] E. Vlieg, M. Deij, D. Kaminski, H. Meekes, and W. van Enckevort, Faraday Discuss. 136, 57 (2007).

[5] G. Meng, N. Arkus, M. P. Brenner, and V. N. Manoharan, Science 327, 560 (2010).

[6] C. R. A. Catlow, S. T. Bromley, S. Hamad, M. Mora-Fonz, A. A. Sokol, and S. M. Woodley, Phys. Chem. Chem. Phys. 12, 786 (2010).

[7] S. Karthika, T. K. Radhakrishnan, and P. Kalaichelvi, Crystal. Growth Des. 16, 6663 (2016).

[8] M. Holmes-Cerfon, Annu. Rev. Condens. Matter Phys. 8, 77 (2017).

[9] P. R. Rowland, Discuss. Faraday Soc. 5, 364 (1949).

[10] P. R. Unwin, Faraday Discuss. 136, 409 (2007).

[11] A. R. Oganov and C. W. Glass, J. Chem. Phys. 124, 244704 (2006).

[12] C. P. Massen and J. P. K. Doye, Phys. Rev. E 75, 037101 (2007).

[13] D. J. Wales, Chem. Phys. Chem. 11, 2491 (2010).

[14] A. R. Oganov, A. O. Lyakhov, and M. Valle, Acc. Chem. Res. 44, 227 (2011).

[15] F. Calvo, J. P. K. Doye, and D. J. Wales, Nanoscale 4, 1085 (2012).

[16] D. J. Wales, J. Chem. Phys. 142, 130901 (2015).

[17] B. W. van de Waal, J. Chem. Phys. 90, 3407 (1989).

[18] C. L. Cleveland and U. Landman, J. Chem. Phys. 94, 7376 (1991).

[19] B. W. van de Waal, Phys. Rev. Lett. 76, 1083 (1996).

[20] J. P. K. Doye, D. J. Wales, and R. S. Berry, J. Chem. Phys. 103, 4234 (1995).

[21] B. W. van de Waal, G. Torchet, and M. F. de Feraudy, Chem. Phys. Lett. 331, 57 (2000).

[22] N. V. Krainyukova, R. E. Boltnev, E. P. Bernard, V. V. Khmelenko, D. M. Lee, and V. Kiryukhin, Phys. Rev. Lett. 109, 245505 (2012).

[23] P. Schwerdtfeger, N. Gaston, R. P. Krawczyk, R. Tonner, and G. E. Moyano, Phys. Rev. B 73, 064112 (2006).

[24] N. V. Krainyukova, Eur. Phys. J. D 43, 45 (2007).

[25] P. Schwerdtfeger, R. Tonner, G. E. Moyano, and E. Pahl, Angew. Chem. Intl. Ed. Engl. 55, 12200 (2016).
[26] H. Cox, R. L. Johnston, and J. N. Murrell, J. Solid State Chem. 145, 517 (1999).

[27] Y. Shibuta, K. Oguchi, T. Takaki, and M. Ohno, Sci. Rep. 5, 13534 (2015).

[28] C. Leitold and C. Dellago, J. Chem. Phys. 145, 074504 (2016).

[29] M. B. Sweatman and L. Lue, J. Chem. Phys. 144, 171102 (2016).

[30] R. J. Baxter, J. Chem. Phys. 49, 2770 (1968).

[31] S. B. Yuste and A. Santos, Phys. Rev. E 48, 4599 (1993).

[32] T. W. Cochran and Y. C. Chiew, J. Chem. Phys. 124, 224901 (2006).

[33] M. Holmes-Cerfon, S. J. Gortler, and M. P. Brenner, Proc. Natl. Acad. Sci. USA 110, E5 (2013).

[34] N. Arkus, V. N. Manoharan, and M. P. Brenner, Phys. Rev. Lett. 103, 118303 (2009).

[35] N. Arkus, V. Manoharan, and M. Brenner, SIAM J. Discrete Math. 25, 1860 (2011).

[36] R. S. Hoy, J. Harwayne-Gidansky, and C. S. O'Hern, Phys. Rev. E 85, 051403 (2012).

[37] R. S. Hoy, Phys. Rev. E 91, 012303 (2015).

[38] M. C. Holmes-Cerfon, SIAM Rev. 58, 229 (2016).

[39] Y. Kallus and M. Holmes-Cerfon, Phys. Rev. E 95, 022130 (2017).

[40] K. Schütte and B. L. van der Waerden, Math. Ann. 125, 325 (1952/53).

[41] J. H. Conway and N. J. A. Sloane, Sphere Packings, Lattices and Groups, Grundlehren der mathematischen Wissenschaften Vol. 290 (Springer Science \& Business Media, New York, 2013).

[42] K. Bezdek and S. Reid, J. Geom. 104, 57 (2013).

[43] P. Erdős, Am. Math. Mon. 53, 248 (1946).

[44] F. H. Stillinger, Phys. Rev. E 59, 48 (1999).

[45] Y. Forman and M. Cameron, J. Stat. Phys. 168, 408 (2017).

[46] S. Heiles and R. L. Johnston, Int. J. Quantum Chem. 113, 2091 (2013).

[47] J. W. R. Morgan, D. Mehta, and D. J. Wales, Phys. Chem. Chem. Phys. 19, 25498 (2017).

[48] P. Jerabek, O. Smits, E. Pahl, and P. Schwerdtfeger, Mol. Phys. 116, 1 (2018).

[49] J. P. K. Doye, M. A. Miller, and D. J. Wales, J. Chem. Phys. 111, 8417 (1999).

[50] P. A. Braier, R. S. Berry, and D. J. Wales, J. Chem. Phys. 93, 8745 (1990).

[51] D. J. Wales, Science 293, 2067 (2001). 
[52] D. J. Wales, Energy Landscapes: Applications to Clusters, Biomolecules and Glasses (Cambridge Molecular Science, Cambridge, 2004).

[53] "Pele: Python energy landscape explorer", https://github.com/ pele-python/pele (2017).

[54] Z. Li and H. A. Scheraga, Proc. Natl. Acad. Sci. USA 84, 6611 (1987).

[55] D. J. Wales and H. A. Scheraga, Science 285, 1368 (1999).

[56] D. J. Wales and J. P. K. Doye, J. Phys. Chem. A 101, 5111 (1997).

[57] J. P. K. Doye and D. J. Wales, J. Chem. Phys. 116, 3777 (2002).

[58] D. E. King, J. Mach. Learn. Res. 10, 1755 (2009).

[59] D. C. Wallace, Phys. Rev. E 56, 4179 (1997).

[60] F. Sciortino, W. Kob, and P. Tartaglia, Phys. Rev. Lett. 83, 3214 (1999).

[61] M. R. Hoare and P. Pal, Adv. Phys. 24, 645 (1975).

[62] M. R. Hoare and J. McInnes, Faraday Discuss. Chem. Soc. 61, 12 (1976).

[63] M. R. Hoare, Adv. Chem. Phys. 40, 49 (1979).
[64] We define spheres that have an equilibrium distance between 0.9 and 1.1 to be bound.

[65] A. L. Mackay, Acta Crystallogr. 15, 916 (1962).

[66] J. Uppenbrink and D. J. Wales, J. Chem. Soc. Faraday Trans. 87, 215 (1991).

[67] C. L. Phillips, E. Jankowski, M. Marval, and S. C. Glotzer, Phys. Rev. E 86, 041124 (2012).

[68] A. Hermann, M. Lein, and P. Schwerdtfeger, Angew. Chem. Intl. Ed. Engl. 46, 2444 (2007).

[69] N. Kaltsoyannis, Angew. Chem. Intl. Ed. Engl. 56, 7066 (2017).

[70] T. D. Della and C. H. Suresh, Phys. Chem. Chem. Phys. 18, 14588 (2016).

[71] P. Schwerdtfeger, L. N. Wirz, and J. Avery, Wiley Interdisc. Rev.: Comput. Mol. Sci. 5, 96 (2015).

[72] Á. González, Math. Geosci. 42, 49 (2010).

[73] B. Keinert, M. Innmann, M. Sänger, and M. Stamminger, ACM T. Graphic. 34, 193 (2015).

[74] A. Kratzer, Z. Phys. 3, 289 (1920).

[75] O. R. Musin and A. S. Tarasov, Discrete Comput. Geom. 48, 128 (2012). 